\title{
Optimization of agroecological efficiency of transport processes in agriculture
}

\author{
Serhii Kharchenko ${ }^{1}$, Oksana Pankova ${ }^{1 *}$, Mikhail Shulyak ${ }^{1}$, Alexander Anikeev ${ }^{1}$, and Kirill \\ Sirovitskiy ${ }^{1}$ \\ ${ }^{1}$ P. Vasilenko Kharkov National Technical University of Agriculture, 44, Alchevskykh street, \\ Kharkiv, 61002, Ukraine
}

\begin{abstract}
Agricultural production is dispersed in huge areas and requires the transfer of a large number of technological and operational materials, intermediate and final products. Therefore, in the agricultural sector, transport occupies an important place: it begins and completes the processes of crops production and livestock products and carries out technological connections between individual states of work. With the development of agricultural production, the role of transport is steadily increasing: if at present, at the average $45 . .50$ tons of different cargoes is the share of each hectare of ploughed field, then in the coming years, an increase of this volume is expected. For more than $35 \%$ of the volume of the main work types is the share of transport works which are carried out by tractors in the agrarian sector. Increasing the dynamic and energy indices of tractors during transport operations in the agrarian sector by ensuring their functional stability will lead to lower energy consumption, increase the ecological efficiency of transport processes and will ensure a steady increase in profits in the agro-industry. The concept of the formation of tractor functional stability during transport works, which is based on the reduction of amplitudes of accelerations (slowdowns) in a three-dimensional geometric space, which, unlike the known ones, provides reduction of additional energy costs, is substantiated in the article.
\end{abstract}

\section{Introduction}

In the process of economic development, production processes that are focused on providing different market needs, in particular, in crop production, are becoming increasingly relevant. Effective implementation of these processes is capable of providing a logistic approach that allows optimizing the entire chain of storage and marketing of agroecological products, ranging from material support to implementation [1-2]. One of the key tasks of the country's food security is to further develop the grain storage sector and ensure the efficient use of transport for its timely delivery to its target. All this can be achieved by introducing elements of optimization of agroecological logistics processes. The assessment of export opportunities of the Ukrainian agroindustrial complex has shown the necessity of expanding and optimizing the transport sector to meet the needs of production and

\footnotetext{
* Corresponding author: pankova oksana@ukr.net
} 
increasing the economic effect. The constant increase in agroecological requirements for the quality of products compels manufacturers to look for new pressing methods of storage and transportation [3-4]. The need for implementation of modern methods and optimization of existing ones was formulated by N. Prisiyazhniyk [5]. Modern European experience shows that ensuring the production of modern scientifically-based methods increases the level of profitability by $15-25 \%$ and it is one of the most effective vectors for the development of wholesale trade, which opens the prospects for the development of the agrarian sector and improves the quality results for the fulfillment of food targets [6-7]. The selection of rational criteria for the delivery of agricultural cargoes plays an important role in ensuring the marketing of products and rationalization of grain transportation technologies, which are not possible without the introduction of computer technologies and modern methods of calculating operating costs [8-10]. Also, an important role is played by agroecological quality control of products. One of the practical ways to increase the effectiveness of this control is to make it in a recycling scheme when a "qualitative object" is checked again [11].

\section{Methodology}

Based on the review of scientific and technical literature on the use of tractors during transport works in the agricultural sector, outstanding issues were identified (including: functional stability, energy conservation, and dynamics).

In order to increase the efficiency of tractor-trailer trains and the stability of their operation, various constructive solutions to this problem were proposed. For example, V. Shalyagin and V. Yasenevich proposed application of active drive wheels of the trailer to increase the pull and energy efficiency indicators of the tractor-trailer train. At the same time in the work of these scientists it was pointed to the need to justify the parameters of functional stability of tractors during transport works. For tractor-trailer trains Z. Zebelyshynsky recommended to take as the main functional parameter the motion roadholding, taking into account the effect of the distribution of propelling force between the axles of the tractor on resistance to skidding (jack-knifing).

The prospect of applying information about the acceleration of mobile machines in evaluating their functional parameters was substantiated. By analogy with related branches of science and technology, the functional stability of transport aggregates can be estimated according to their functional stability, which characterizes the deviation of the basic parameters of complex technical systems from the nominal values when the accelerated motion and normal motion.

When solving the energy saving problem it is recommended to evaluate the tractor efficiency during the transport work at effective work which is carried out by the transport unit per unit time of the cycle (delivery of cargo to place of consignment, return). In this case, the effective work is determined by the work of the tractor's driving forces, less the work spent on overcoming the forces of harmful resistance. Dedicated to the problem of energy saving of mobile agricultural units, in which it is concluded that there is a interconnection between the energy consumption of these aggregates from the stability of their high-speed motion. This problem for tractor-based transport units has not been resolved.

Different methods and devices are proposed for the estimation of functional stability, energy saving and dynamics of mobile machines in which attention was paid to the lack of control systems and executive system of the functional stability of the tractor operation.

The conception of the dynamic space of TA functioning is formulated, on the basis of which the functional parameters of the tractor during transport works are substantiated. 


\section{Empirical results}

In general, the transport unit (Fig. 1) is represented in the form of a system at the entrance of which there are vector-functions of control $U(t)$ and excitation $F(t)$. Output variables, which characterize the functional parameters of the TA, are represented by a vectorfunction $Y(t)$. Dynamic properties of the TA are characterized by a transfer function $W_{m}=$ $S_{y} / S_{u}$, where, $S_{y}$ and $S_{u}$ - the output and control parameters accordingly.

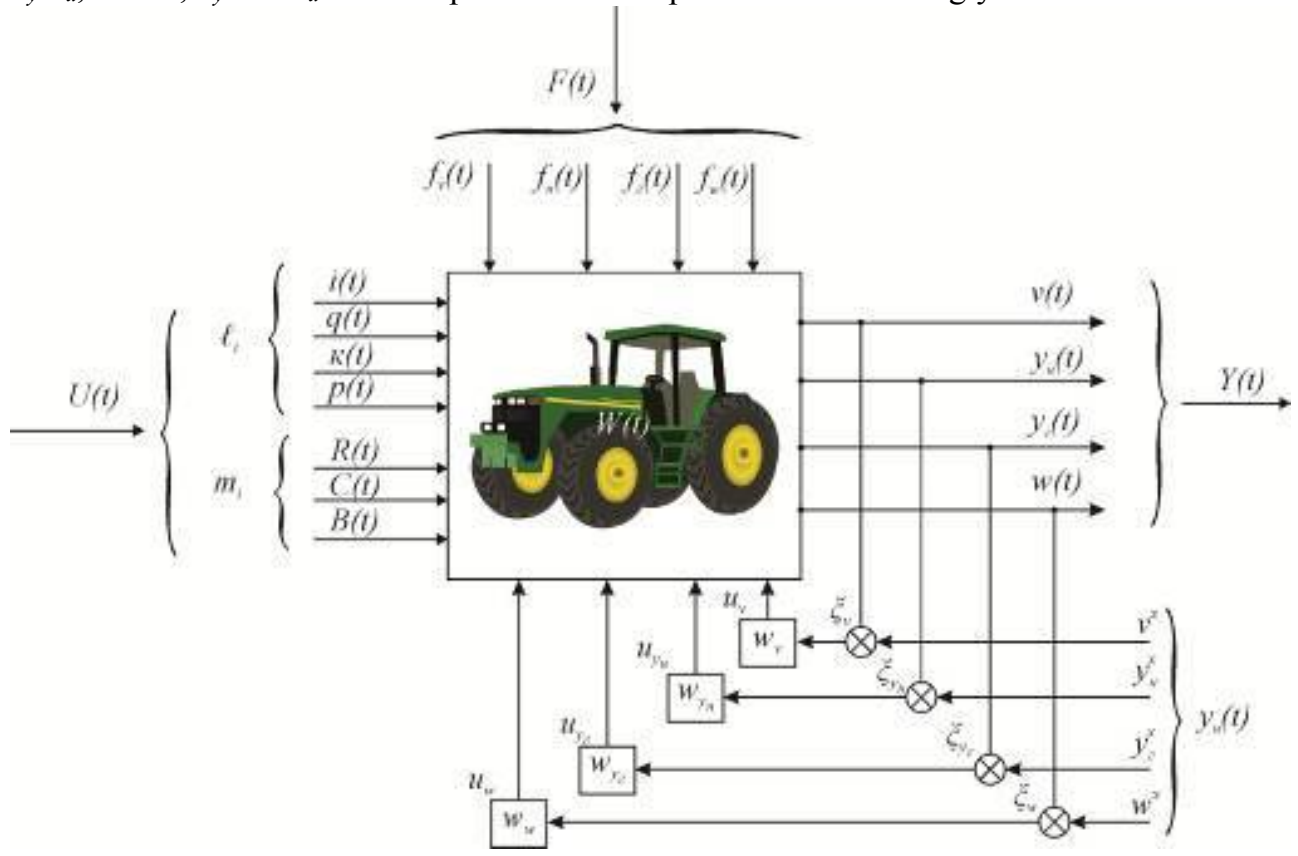

Fig. 1. Generalized functional diagram of the transport unit.

The TA is influenced by both controlled input vector-control functions $l i[i(t)-$ gear ratio, $q(t)$ - fuel supply, $\kappa(t)$ - gear ratio of the steering, $p(t)$ - the brake control effort], and uncontrolled $m_{i}[R(t)$ - motion resistance, $C(t)$ - road relief, $B(t)$ - wind force, etc.]. Output variables $Y(t)$ are characterized by the rate of motion $v(t)$, motion-direction stability $y_{H}(t)$ and braking $y_{2}(t)$, by the stability of the dynamic properties $w(t)$ of the unit.

Usually, excitations $F(t)$ are determined by the instability of the output (functional) parameters $v(t), y_{u}(t), y_{2}(t)$ and $w(t)$. When comparing these parameters with their values $v^{x}$ $y^{x}{ }_{\mu} y^{x}{ }_{2} w^{x}$ and, with which TA functions stably, at the links of the comparison $\xi_{v}, \xi_{H}, \xi_{2}$ and $\xi_{w}$ with the transfer functions $w_{v}, w_{y \mu}, w_{y z}$, and $w_{w}$ the signals $u_{v}, u_{y \mu}, u_{y z}$, and $u_{w}$ of the correction of the controlled input variables $u(t)$ are generated.

The functional parameter $v(t)$ is characterized mainly by the input control functions $i(t)$ and $q(t)$, and determines the TA running qualities. The parameters $y_{H}(t)$ and $y_{2}(t)$ are determined mainly by $v(t)$, excitations of the motion direction $f_{\mathrm{H}}(t)$ and braking $f_{\Gamma}(t)$ and they are aimed at ensuring the motion safety of the TA. The dynamic parameter $w(t)$ characterizes the TA motion in the longitudinal, horizontal and vertical planes under the action of forces in these planes.

The dynamic space of the tractor's operation during transport works is based on the metric space of states, each element of which completely determines the state of the considered system in terms of functional parameters $v(t), y_{H}(t), y_{2}(t)$ and $w(t)$. 
In the case of an unstable one or more of the tractor's functional parameters $v(t)$, $y_{\mathrm{H}}(t), y_{2}(t)$ and $w(t)$, which is characterized by a deviation from the nominal values $y^{x}$, $y_{H}^{x}, y_{2}^{x}$ and $w^{x}$, it is possible the loss of the functional stability tractor. In this case it will not perform the functions, which determined by the normative-technical documentation (NTD). In this case, the dynamic space of the tractor's operation is determined by the transfer functions $W_{v}, W_{y_{\mathrm{H}}}, W_{y_{2}}$ and $W_{w}$, which characterized by the ratio $v(t), y_{\mathrm{H}}(t)$, $y_{2}(t), w(t)$ from $y^{x}, y_{H}^{x}, y_{2}^{x}, w^{x}$.

When operating a tractor during the transport work, the task of ensuring its functioning in one of two areas is solved: OA - the operation area, in which the tractor operates according to the purpose (the necessary traction effort, stability of the driving direction and braking, etc. are provided); $O O$ - area of optimal operation, for example, by the criterion of energy saving, in which the tractor operates with the allowable change of speed (change of movement acceleration within the permissible limits) (fig. 3). In this figure, by the points $B_{y}$ and $B_{g}$ marked accordingly to the tractor operation in the conditions of unsteady mode of operation without acceleration and permissible area according to energy conservation; $\Delta_{v}$ - reserve of optimal functioning; $V_{o}, V_{\phi}$ - the functioning vectors is optimal, actual; $\Delta B$ - reserve of functioning in the area AF.

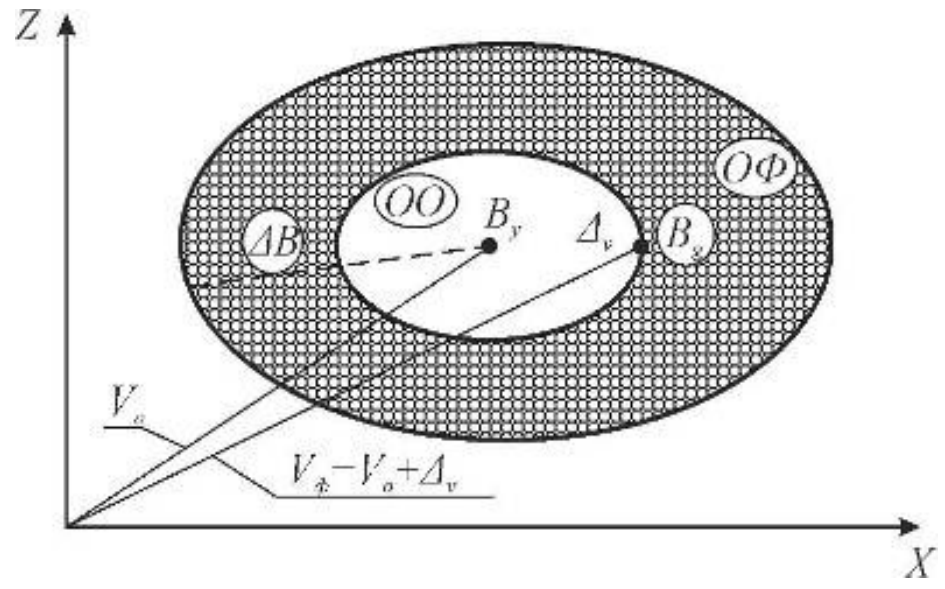

Fig. 2. Correlation of areas of optimal work $O O$ and the tractor operation $O \Phi$.

In order to solve the scientific problem of ensuring the tractor functional stability during transport work, it has been provided the methodology of the choice of the nominal values of the functional parameters $v(t), y_{\mathrm{H}}(t), y_{2}(t), w(t)$ and also the tolerance zone on them, with which the tractor work in the zone $O O$ is substantiated. Based on the fundamentals of theoretical mechanics, the energy analysis of the transport aggregate is carried out during the active and passive tractor operation in the dynamic space of operation. In this case, useful (active) work is determined by the work of the driving forces, less the work spent on overcoming the forces of parasitic drag, which characterizes the noneffective (passive) work of the tractor. The passive work of force is equal to the product of its module on the path and on the cosine of the angle $\alpha$ between the directions of force and movement.

Proposed indicators of tractors functional stability during the transport works, which include: rate of motion $v(t)$, stability of the motion direction $y_{H}(t)$ and braking $y_{2}(t)$, 
stability of dynamic properties $w(t)$. If one or more parameters $v(t), y_{H}(t), y_{2}(t), w(t)$ are unstable, the functional stability of the tractor may be lost. In this case, the problem is solved on the basis of the justified methodology of providing the work of the transport aggregate in the zone of optimal work according to the criterion of energy conservation.

\section{References}

1. V. Melnik, A. Anikeev, K. Sirovitskiy, Eng. of Natural Management 2(8), 6-10 (2017)

2. S. Kornienko, V. Pashenko, V. Melnik, S. Kharchenko, N. Khramov, East.-Europ. JET 5, 34-43 (2016)

3. S. Gritsenko, DonNTU. Series: economic 5, 185-189 (2014)

4. V. Nelep, Economy of Ukraine 9, 54-63 (2011)

5. N. Prysyazhnyuk, P. Sabluk, M. Kropivko, Economy of Ukraine 6, 4-16 (2011)

6. E. Shubravskaya, N. Rindenko, Economy of Ukraine 8, 77-85 (2012)

7. E. Shubravskaya, Economy of Ukraine 8, 64-76 (2013)

8. V. Nefyodov, Yu. Tkachenko, East European Journal of Advanced Technologies 3(63), 13-15 (2013)

9. S. Kharchenko, O. Anikeev, M. Tsiganenko, O. Kalyuzhny, KhNTUSG (MAP) 156, 174-179 (2015)

10. D. Avalos-Gonzalez, D. Hernandez-Balbuena, V. Tyrsa, O. Sergiyenko, F. MurrietaRico, V. Kartashov, M. Kolendovska, S. Sheiko, V. Melnyk, IECON 3159-3164 (2018)

11. V. Puzik, L. Puzik, N. Lyubymova, O. Pankova, V. Sakhatsky, IJET (UAE) 7, 507513 (2018) 